Permit, in conclusion, a single reflection upon the moral aspect of this singular case :

All experience shows, all history proves, from the earliest moment of recorded time, that one of the brightest attributes of human nature is that which prompts respect for the solemn scenes which surround the departure of a human soul. Among all civilized people, particularly among Christian and Hebrew societies, aye! even among pagan nations in modern times, when the wings of the Angel of Death begin to cast their dark shadow upon a household all business is laid aside, passion is hushed, avarice is mute, footsteps are muffled, friends communicate in whispers, silence surrounds the solemn scene. If the ties of blood or affection are about to be severed, tears of affection bedew our cheeks while aspirations of prayer escape our lips. What do we find in this case? No sooner is the alarm given that the dread summons is approaching, than fleet horses are harnessed, a huge document is prepared bearing great seals. The pretended executor of the estate of the dying man, and his unwilling beneficiary to the amount of thousands of dollars, procures a dual signature to a document giving the substantial control of every dollar of his estate to himself; and instead of standing by the bedside of his friend to show sympathy and comfort, he is seen, armed with his commission, flying back and forth to perfect his documents for two full days between Spring Valley and New York, with all the speed which nimblefooted horses and lightning express trains could afford, until we find him knocking at the door of the bankers of the decedent to seize his last dollar at the very moment when the immortal soul of that benefactor was taking its departure to realms unknown. Seldom do the annals of avarice show a more depraved exhibition of human nature.

\section{A NEW FORM OF APPARATUS FOR ADMINISTER- ING GASEOUS ENEMATA.}

Read in the Section on Medicine, Materia Medica and Therapeutics, at the Thirty-Eighth Annual Meeting of the American Medical Association, Fune 7, 1887.

BY J. H. KELLOGG, M.D., OF BATTLE CRERK, Mich.

The phenomenal interest which has been manifested in the so-called Bergeon method of treating pulmonary consumption by means of gaseous enemata, which is by no means confined to the profession, but has been largely shared by the general public, has created a most unprecedented demand for a new form of medical appliance designed to administer gaseous enemata. Thus far this demand has been supplied by the simple and effective device of Dr. Morel. Many of those who have undertaken the extensive use of this apparatus, have experienced great annoyance by reason of the almost utter impossibility of totally excluding atmospheric air from the apparatus. The inaccurate and tedious mode of using it, and the necessity for the handling of corrosive chemicals in the treatment of each indi- vidual case. I encountered all these difficulties at once on beginning the trial of the new method, and was deeply impressed with the importance of designing some more exact and less inconvenient method of administering the treatment. I soon designed and subsequently had constructed the simple apparatus which I herewith exhibit. The special features of the apparatus consist:

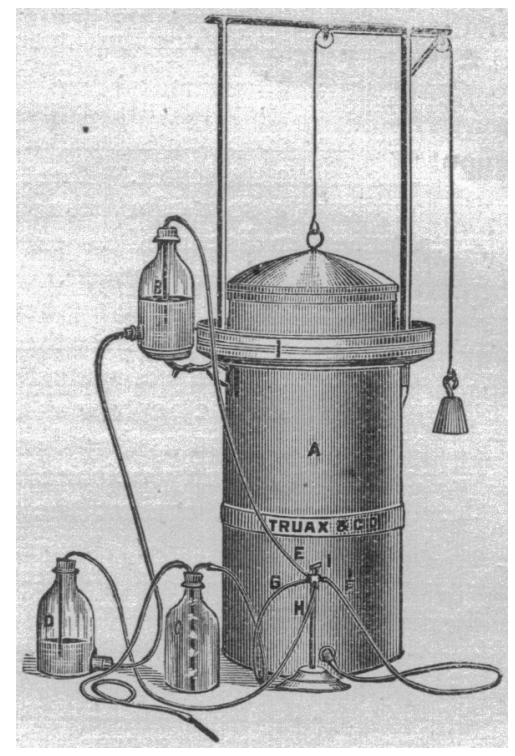

I. Of an ordinary gasometer similar to that used in the Waldenberg apparatus for the treatment of pulmonary diseases.

2. Of 2 three-necked Wolffe's bottles. The 2 bottles are connected by means of glass and rubber tubes. The 2 wash bottles are connected thus: The perforated rubber stopper of the middle neck of each bottle has fitted into it a glass tube of sufficient length to reach to within a quarter of an inch of the bottom of the bottle. These 2 tubes are connected by a continuous tube. The corresponding side necks of the 2 bottles are connected by means of rubber tubes attached to glass tubes, in the perforated stoppers of just sufficient length to reach through the stopper. Each end of each of these tubes is supplied with a stop-cock, such as is used with the ordinary fountain syringe. Each tube is divided in the center, and each end connected to the opposing ends of a brass tube made in the form of a T. To these T's are connected, on one side, a tube leading to the gasometer or storage tank, and on the other, a rubber tube leading to a wash bottle containing water charged with sulphuretted hydrogen, as in the apparatus now in use, from which the mixed gases are conveyed to the patient in the usual way.

To prepare the apparatus for use we have only to fill the gasometer with carbon dioxide and one of the Wolffe bottles with water, and all is in readiness. Pressure is obtained by elevating the Wolffe bottle which is filled with water, to a height of 12 to 20 inches. After elevating the bottle, open the stopcock controlling the connection between the upper bottle and the tank, and also the stop-cock controlling 
the connection between the lower bottle and the mixing bottle. The water in the upper bottle now begins at once to syphon into the lower bottle, and in so doing displaces the contents of the lower bottle while the upper bottle fills with $\mathrm{CO}_{2}$ from the tank. When the upper bottle is empty, it is only necessary to close the 2 stop-cocks, reverse the positions of the bottles, and open the opposite corresponding stopcocks, and the same action will be repeated.

This apparatus is, of course, designed especially for office use. From what I have seen and learned of this method of treatment, I am inclined to believe that it is not by any means prudent to leave it in the hands of an ordinary nurse. A thoroughly trained nurse could, undoubtedly, learn to administer the treatment as well as a physician, and might safely be trusted with the treatment in individual cases, as has been suggested; but educated nurses are not abundant, and certainly ordinary nurses, which are the only ones accessible in the majority of cases, except in a few of the larger cities, are wholly incompetent to administer a remedy which has produced death in lower animals in so small doses as $I$ and $y / 2$ cubic inch. I am also of the opinion that the treatment may be much more successfully carried out in an office practice than at the bedside, except, of course, in cases in which the patient is so reduced by disease as to be confined to his bed or his room. For cases of the sort last mentioned, the apparatus which I am describing cannot, of course, replace the Morel apparatus. But I am confident that for office use it will be found very much superior to the Morel apparatus in at least the following particulars:

I. Convenience. A storage tank may be employed of any size desired. The tank which I here exhibit holds ro gallons and cost less than $\$ 6$. The carbon dioxide may be made at any convenient time and may be kept in a tank without deterioriation for almost any length of time.

Another element of convenience is found in the fact that when set going the apparatus is nearly automatic. The Wolffe bottles which I show here hold about I litre each, and consequently it is necessary to make but $\mathrm{I}$ change in the apparatus for each litre of gas employed, whereas, in the old apparatus, the physician must keep his hand continually upon the injecting bulb and give undivided attention to the management of the apparatus. By the employment of bottles holding $\mathrm{r}$ gallon or more, the number of attachments of the apparatus necessary in a given treatment may be proportionately lessened.

2. Comfort of the patient. There has been much complaint of pain caused by the employment of gaseous enemata, and patients have in numerous instances discontinued the treatment on this account, who might possibly have been benefited if the treatment could have been continued. The chief cause of pain is generally believed to be the injection of air, or the too rapid administration of the gas. It is almost impossible to effect the injection of small quantities of air in the use of the Morel apparatus. It is impossible to roll the rubber bag so tight that every particle of air will be excluded, and the greatest care is necessary to prevent the inclusion of so great a quantity of air as to give the patient much discomfort. It is also not at all easy to maintain a constant or uniform flow of gas by means of an in. jecting bulb, which is necessarily intermittent. This new apparatus wholly obviates both of these difficulties. When the storage tanks and the 3 bottles employed are once filled with carbon dioxide it is impossible for any air to find access to the apparatus or to be injected.

In filling the storage tank, the total exclusion of air may be secured by simply sinking the inner cylinder until it is wholly filled with water, while the amount of air in the wash bottles is so small that a single filling and emptying of each of the Wolffe bottles is sufficient to exclude it entirely. The advantage which this apparatus presents over the Morel apparatus in this particular is as great as that presented by the fountain syringe over the old-fashioned bulb or piston syringe. In the employment of this apparatus, the flow of gas is necessarily uniform, or so nearly so that no perceptible change could possibly be observed by the patient. The rate of flow may be regulated to a nicety by the elevation or lowering of the bottle which is filled with water. Any degree of pressure desired may be obtained. I have found an elevation of 20 inches to be sufficient to overcome the pressure of gas within the bowels. The time required for the injection of a litre of gas by this apparatus, the acting bottle being raised to a height of 20 inches, is from 2 to 3 minutes; with an elevation of 10 inches, the action is considerably slower. The flow of gases may be instantly stopped at any moment by the closure of either one of the 2 stop-cocks, which are also open. When the apparatus is in action, the patient or physician may stop the flow of gas instantly by simply depressing the conducting or injecting tubes.

3. Accuracy. In the employment of so powerful a toxic agent as sulphuretted hydrogen, it must be evident that the matter of precise and carefully regulated doses is just as important as in the employment of arsenic, strychnia, morphia, or other agents capable of producing poisonous as well as therapeutic effects.

A rubber bag and an injecting bulb can hardly be regarded as the equivalent of the balance and the graduated burette; but by means of this apparatus, the amount of carbon dioxide injected may be measured with the greatest precision. It is only necessary to graduate the Wolffe bottles by which the flow of gas is actuated, and by noticing the amount of liquid moved from one bottle to the other, the precise amount of gas which has been injected may be ascertained. In this respect, the apparatus seems to leave nothing to be desired. The liquid employed in the bottles is simply water colored with a little analine to render the movements of the liquid more perceptible. As presented here, the apparatus is in a somewhat crude form as it is the first one which I have constructed, and a more complete apparatus which I had ordered made was not completed in time to present to this meeting. Nevertheless, I have been employing it for some little time, and have been much pleased with its performance. 
One patient to whom the Morel apparatus invariably gave a very considerable amount of pain, notwithstanding the greatest care in its use, receives the treatment from this apparatus without the slightest discomfort.

I think the use of Wolff's bottles with but 2 necks and an opening at the bottom of the bottle will be found more convenient than the form which I am now using, as it will obviate the use of the syphon which fails to operate without tilting the bottle after the column of water has been broken by allowing the upper bottle to become completely empty. I have also devised a more convenient and substantial arrangement of the stop-cocks which I am having constructed.

I do not offer this apparatus as one which must necessarily supplant the Morel apparatus, but for office use and for careful scientific investigation it offers the elements of convenience to the physician, comfort to the patient and accuracy of dosage, ad. vantages which must be at once appreciated by those who are at all familiar with the administration of this new mode of treatment.

\section{AIDS IN THE PREVENTION OF FEVERS.}

\author{
Abstract of a Paper read before the Section on Practice of Med. \\ icine, Materia Medica and Physiology, at the Thirty \\ Eighth Annual Meeting of the American Medical \\ Association, Fune, 1887 . \\ BY R. W. SEAY, M.D., \\ OF PILCHER'S POINT, LA.
}

In July, 1883, I was called to see L. G. L., who was suffering from a severe attack of remittent fever, from which he had not been free for the past seven days. By a judicious course of treatment his fever was abated by noon next day. I called in the evening, and as I was cautioning him to take sulphate of quinine as I directed, and that if, in spite of the treatment, the fever should return, he must take a fluid mixture I left to be taken during the existence of the fever, he said: "Can't I take some of the fever mixture whether the fever is on me or not?" I thought a moment, and as I looked upon his pale, emaciated face and knew the disastrous effects a return of fever would have upon him, and as I hoped to be able to see him again in six or eight hours, I told him to take the mixture just as he had taken it while the fever was upon him. He was also directed to keep up the quinine. He had no return of fever for weeks.

His query and the beneficial effects of the treatment pursued made me reflect on the use more often, as preventatives, of those remedies which we use during the pyrexia. I have since tried this experiment in more than roo cases, and in every case in which the test was properly carried out, there was no return of fever. I desire that you should understand that I allude more particularly to intermittent malarial fevers, but $I$ think the day is not distant when this form of treatment can be beneficially used in obstetric and surgical cases.

In recapitulation of the causes of fever we will say they are: r. Arterial contraction.

2. Accelerated action of heart.

3. Increased tissue metamorphosis or oxidation.

4. Probably a nervous influence causing an inhibitory influence on the usual changes in transformation.

If we can give remedies which will prevent the three first known causes mentioned and the last the. oretical cause, we can lessen or entirely prevent the occurrence of fever, in many cases. We all use these remedies in the pyrexia, and we can advantageously use them in the preventive treatment. We have such remedies in veratrum viride, aconite and similar remedies. Veratrum viride acts upon the cardiac ganglia and the muscular substance of the heart, as is proved by paralyzing the pneumogastric with atropia, and it still affects these parts. Aconite, we are told, acts in the same way. I do not mean, in using these remedies, to prohibit the use of quinine, but that their proper use, as suggested, as aids in the prevention of fevers. Use, for instance, for an adult not very weak, this prescription:

R. Tr. veratri viridi (Norwood's)....... Mxxxij. Tr. aconitii................... $m_{x \times x i j}$ Spts. ætheris nitrosi. ............. ziv.

Aqux q. s. ad..................

m.

S. Give, in a half-wineglass of water, a teaspoonful every 3 hours, beginning 9 hours before the expected return of fever, and discontinue after fever time has passed.

I desire to impress upon you that I wish to recommend medicine which will prevent the four causes which I have summed up as producing fever, rather than recommend any particular medicines, for we may have our own preference for several remedies, either of which may produce the same effect. One may prefer, for instance, veratrum viride to either aconite or gelsemium, while another may prefer to use aconite in preference to any of the other cardiac sedatives. I wish the principle to be ever present to your minds, and then you have your choice of several varieties of prescriptions.

\section{ATHEROMA OF THE LEFT CORONARY ARTERY RE- SULTING IN ANEURISM OF THE APEX OF THE LEFT VENTRICLE.

\author{
Read before the Chicago Medical Society, Fune 6, 1887, \\ BY ROBERT TILLEY, M.D., \\ of chicago.
}

As this subject is so little associated with my usual practice it is with some hesitancy that I present even this short report. Aneurisms of the heart, however, are not common in clinical reports, and I trust that fact alone will justify the presentation of the case.

The patient was an intimate friend of mine, and it was only at his special request that I took charge of the case. He was 57 years of age, and his occupation was that of a lawyer. His previous life was unexceptionally exemplary. He did not use tobacco in any form, and was exceedingly temperate and methodical in all his habits; his carriage was such as 